\title{
An assessment of vertigo patients presenting to the otorhinolaryngology and neurology outpatient clinics
}

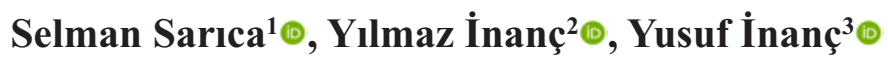 \\ ${ }^{1}$ Department of Ear, Nose and Throat, Kahramanmaraş Sütçü Imam University School of Medicine, Kahramanmaraş, Turkey \\ ${ }^{2}$ Department of Neurology, Kahramanmaraş Sütçü Imam University School of Medicine, Kahramanmaraş, Turkey \\ ${ }^{3}$ Department of Neurology, Gaziantep University School of Medicine, Gaziantep, Turkey
}

DOI: $10.18621 /$ eurj.408135

\begin{abstract}
Objective: Vertigo is a general term used for disorientation and is a frequent cause of admission to emergency services, otorhinolaryngology and neurology outpatient clinics. This study aims to evaluate the clinical findings, diagnostic laboratory tests, brain magnetic resonance imaging, and Doppler ultrasonography results of patients with complaints of vertigo.

Methods: Patients aged $>18$ years who admitted to the Otorhinolaryngology and Neurology Outpatient clinics of Kahramanmaraş Sütçü İmam University Training and Research Hospital with the main complaint of vertigo between January 2017 and August 2017 were retrospectively reviewed. The patients were evaluated in respect of anamnesis, examination findings, and diagnostic tests (pure tone audiometry, video nystagmography, brain magnetic resonance imaging, and carotid and vertebral artery Doppler ultrasonography).

Results: The study included a total of 101 patients with a mean age of $46.36 \pm 16.1$ years who presented with the complaint of vertigo. Of the patients, 16 had an ischemic gliotic region, 4 had a lacunar infarct, 2 had an arachnoid cyst, 2 had a cerebellar infarct, 1 had a meningioma, and 1 had a pineal gland cyst on the brain magnetic resonance imaging. Of the patients evaluated in the Otorhinolaryngology outpatient clinic, 87 $(86.13 \%)$ were diagnosed with peripheral vertigo and 14 (13.6\%) with central vertigo. A diagnosis of central vertigo was made in $23(22.77 \%)$ patients evaluated in the Neurology outpatient clinic.

Conclusions: For patients presenting with the complaint of vertigo to the otorhinolaryngology and neurology outpatient clinics, a detailed anamnesis and a physical examination precede specific vestibular tests in the diagnosis. A coordinated communication of both clinics is important for a rapid diagnosis and prevention of unnecessary examinations.
\end{abstract}

Keywords: Vertigo, clinical evaluation, magnetic resonance imaging, Doppler ultrasonography, pure tone audiometry, video nystagmography

Received: March 20, 2018; Accepted: May 17, 2018; Published Online: September 3, 2018

V ertigo, which is the general term used for disorientation, is a frequent cause of admission to otorhinolaryngology and neurology outpatient clinics. Patients reporting vertigo have complaints such as the feeling of dizziness, sudden darkening of vision and imbalance [1-3]. The etiology of these symptoms is multifactorial and may include anemia, psychiatric disorders, hypoglycemia, drug effects, cardiovascular diseases and peripheral and central vestibular system disorders $[4,5]$. Vertigo (real dizziness) is defined as

Address for correspondence: Yılmaz Inanç, MD., Kahramanmaraş Sütçü Imam University School of Medicine, Department of Neurology, Kahramanmaraş, Turkey 
the illusion of movement which is not real, where the patient feels that they or the surroundings are turning [6]. Vertigo occurs with an asymmetrical involvement of any of the peripheral or central vestibular pathways of the vestibular system [7]. It should be focused on the vestibular system in every patient with a rotation anamnesis [6].

Vertigo is a symptom that should be attached importance because it affects a wide section of the population up to $20 \%-30 \%$, causes loss of workforce, and can be a symptom of illnesses that may be lifethreatening $[3,8,9]$. In studies conducted on emergency departments, it is reported that the rate of patients presented to emergency departments vary between $3 \%$ and $6.7 \%[2,8,10,11]$. In the evaluation of patients presenting with the complaint of vertigo, there is a need for various radiological and vestibular tests in addition to the anamnesis and physical examination. The main aim is to determine the etiology by differentiating whether the disease is of peripheral or central origin, to establish a diagnosis, and to apply a treatment $[12,13]$. The physician should reach the correct diagnosis by correctly interpreting the anamnesis, and physical examination and test results and combining these with the symptoms of the patient. Despite all efforts of the clinician, difficulties may be experienced in the diagnosis and the determination of whether the disease is peripheral or central in some patients presenting with vertigo. In this period, patients tend to consult more than one physician for their diseases. This study aims to compare the clinical findings, diagnostic laboratory tests and imaging results of patients presenting to the Otorhinolaryngology and Neurology outpatient clinics with the complaint of vertigo.

\section{METHODS}

Patients aged $>18$ years who admitted to the Otorhinolaryngology and Neurology Outpatient clinics of Kahramanmaraş Sütçü İmam University Training and Research Hospital with the main complaint of vertigo between January 2017 and August 2017 were retrospectively reviewed. The approval for the study was granted by the Ethics Committee of Kahramanmaraş Sütçü İmam University School of Medicine in the meeting dated
06.12.2017 (session no:2017/04, decision no: 20). Patients with a neuropsychiatric disorder, systemic disease, history of chronic drug use or a diagnosis of malignancy were excluded from the study.

Detailed neurological and otorhinolaryngology examination findings were recorded and compared. Detailed diagnostic tests applied in respect of the etiology (pure tone audiometry, video nystagmography, brain magnetic resonance imaging (MRI), and carotid and vertebral artery Doppler ultrasonography) were reviewed. The patients were divided into groups according to the diagnosis as peripheral, central, and undefined on the basis of the clinical evaluations in the Otorhinolaryngology and Neurology outpatient clinics. Patients were treated according to the etiology.

The Dix-Hallpike maneuver was performed on patients applied to the Otorhinolaryngology outpatient clinic. The acetylsalicylic acid treatment was initiated in patients with stroke etiology in the Neurology outpatient clinic.

\section{Statistical Analysis}

The statistical analyses of the study data were made using the IBM SPSS for Windows Version 21.0 program. Numerical variables were reported as the mean \pm standard deviation (SD) and the minimum and maximum values. Categorical variables were stated as number (n) and percentage (\%). Before the comparison of the groups in terms of numerical variables, the parametric test assumptions were checked (conformity to normal distribution and homogeneity of variance). The diagnostic and symptom groups were compared in respect of age using the One-Way Variance Analysis. Paired comparisons were made with the Tukey's HSD test. Whether there is a difference between the groupsin respect of categorical variables or not was examined using the Chi-square test. A value of $p<0.05$ was accepted as statistically significant.

\section{RESULTS}

A total of 101 patients admitted to the Otorhinolaryngology and Neurology Outpatient clinics with the complaint of vertigo. The patients comprised of $35(31.65 \%)$ males and $66(65.35 \%)$ 
Table 1. Peripheral and central distribution of patients evaluated in otolaryngology and neurology outpatient clinics

\begin{tabular}{lcc}
\hline & $\begin{array}{c}\text { Peripheral vertigo } \\
\text { n (\%) }\end{array}$ & $\begin{array}{c}\text { Central vertigo } \\
\text { n (\%) }\end{array}$ \\
\hline ENT & $87(86.13 \%)$ & $14(13.6 \%)$ \\
Neurology & $78(78.23 \%)$ & $23(22.77 \%)$ \\
\hline ENT $=$ Otorhinolary
\end{tabular}

ENT $=$ Otorhinolaryngology, $\mathrm{n}=$ the number of the patient

females with a mean age of $46.36 \pm 16.1$ years (range, 18-80 years). Of the patients evaluated in the Otorhinolaryngology outpatient clinic, 87 (86.13\%) were diagnosed with peripheral vertigo and 14 (13.6\%) with central vertigo. The Dix-Hallpike maneuver was applied to all the patients and a positive response was obtained in $72(71.28 \%)$. Of the patients, 5 were diagnosed with Meniere's syndrome, 4 with vestibular neuritis, 5 with otosclerosis, and 1 with labyrinthitis. Grading in saccade that supports pathology, saccadic pursuit, optokinetic asymmetry, and vertical nystagmus that change direction with position were observed in 14 patients. Vertigo had begun suddenly in $89 \%$ and insidiously in $11 \%$ of the patients. A diagnosis of peripheral vertigo was made in $92 \%$ of the patients with sudden onset and in $50 \%$ of those with insidious onset. Chronic otitis was determined in 12 patients, and diplopia and dysarthria in 6 patients. Complaints of diplopia and paresthesia were present in 4 patients. In the hearing evaluation of the right ear, 73 patients were within the normal range at $\angle 25 \mathrm{~dB}$ and a hearing loss was determined at a very mild level (26-40 dB) in 11 patients, mild (41$55 \mathrm{~dB}$ ) in 1 patient, and at an advanced level (56-70 $\mathrm{dB}$ ) in 2 patients (Table 1). In the evaluation of the left ear, 68 patients were normal and a hearing loss was determined at a very mild level in 14 patients, mild in 4 patients, and moderate in 4 patients. Of the 12

Table 2. Lesions detected in MRI

\begin{tabular}{lc}
\hline Detected lesion & $\mathbf{n ~ ( \% )}$ \\
\hline Ischemic gliotic region & $16(15.8)$ \\
Lacunar infarct & $4(3.9)$ \\
Arachnoid cyst & $2(1.9)$ \\
Cerebellar infarct & $2(1.9)$ \\
Meningioma & $1(0.9)$ \\
Pineal gland cyst & $1(0.9)$ \\
\hline
\end{tabular}

$\mathrm{n}=$ the number of the patient patients with chronic otitis, 1 was diagnosed with labyrinthitis and 11 were diagnosed with benign paroxysmal positional vertigo (BPPV).

Physical and neurological examinations were applied to all the patients evaluated in the neurology outpatient clinic. Central vertigo was diagnosed in 23 $(22.77 \%)$ patients and peripheral vertigo in 78 $(78.23 \%)$ (Table1). MRI was performed at an external center or in the Emergency Dept. Of the patients, 16 had an ischemic gliotic region, 4 had a lacunar infarct, 2 had an arachnoid cyst, 2 had a cerebellar infarct, 1 had a meningioma, and 1 had a pineal gland cyst on the brain magnetic resonance imaging (Table 2). The carotid vertebral Doppler USG tests were normal in these patients. Psychiatric causes, orthostatic hypotension and anemia were observed in $40 \%-45 \%$ of the patients presenting at both outpatient clinics.

\section{DISCUSSION}

Vertigo is most often caused by a dysfunction in the vestibular system from a peripheral or central lesion. Patients apply to many outpatient clinics such asemergency department, and otorhinolaryngology, neurology, and internal medicine outpatient clinics with the complaint of vertigo. As one of the most frequently seen complaints in the general population, vertigo has an extremely negative effect on the quality of life $[3,6,14]$. The frequencies of vertigo were $15 \%$ and $12 \%$, respectively, in patients presenting to the otorhinolaryngology and neurology outpatient clinics. About $40 \%-45 \%$ of the patients had complaints such as psychiatric disorders, orthostatic hypotension, and anemia. The present study has similar data.In the literature, vertigo has been reported to be more frequent in females $[13,14]$. In the present study group, vertigo was more commonly (66.35\%) observed in the female gender. Central vertigo was more frequent $(56.52 \%)$ in males, which can be explained by males having more risk factors for central etiologies [15]. In population-based studies on the etiology of vertigo, peripheral vestibular dysfunction has been reported in $40 \%$, central causes in $10 \%$, psychiatric causes in $15 \%$, other reasons in $25 \%$, and undetermined diagnosis in $10 \%$ of patients presenting at the emergency department and first stage outpatient clinics $[16,17]$. In the present study, which 
was more specific as it only included patients presenting to the Otorhinolaryngology and neurology outpatient clinics with the complaint of vertigo, peripheral causes were seen to be the primary cause, which was consistent with the literature. Patients with psychiatric causes were referred to the relevant departments in the neurology or otorhinolaryngology department at the first visit. Therefore, this study had no cases of vertigo due to psychiatric reasons. The central vertigo ratios in both departments were found to be $13.6 \%$ and $23 \%$, respectively. The ratios in the neurology department were slightly higher than rates reported in the literature. The difference in the rate of peripheral and central vertigo in the same patient group presenting to the Otorhinolaryngology and neurology outpatient clinics was found to be a central rate of $13.6 \%$ in the otorhinolaryngology and $23 \%$ in neurology outpatient clinics and this could be attributed to a false negative on the diffusion-weighted MRI in patients with a cerebellar lesion or a brain stem lesion of small dimensions in the first few hours of ischemic stroke. In addition new clinic findings, this can be explained by the inclusion of the ischemic gliotic areas, developing in some regions of the brain conjunction with age and other risk factors, to the etiology group by the neurology outpatient clinic after making other differential diagnoses [18, 19]. In patients with a migraine, there are complaints of vertigo and imbalance at the rate of $70 \%$ either between or during the attacks. Vertigo alternately occurs with concomitant nausea. Just as it can follow the onset of a headache, it can also start during the recovery period [20]. The patients who presented with migrainous vertigo and peripheral vertigo were first evaluated in the present study. A diagnosis of migrainous vertigo was made in 4 patients with a history of a migraine in both the otorhinolaryngology and neurology outpatient clinics. Previous studies have reported migrainous vertigo betweenthe rates of $0.1 \%$ to $9 \%[21,22]$. Peripheral vertigo can be seen independently of a migraine in migraine patients. The peripheral vertigo was determined in 5 of the 9 patients in the present study with a history of a migraine. While the diagnosis of vestibular neuritis is easy with its typical clinical presentation, it is possible to confuse the pathologies at the level of eighth cranial nerve, vestibular nucleus or brain stem with the vestibular neuritis particularly during the Meniere's disease with recurrent vertigo episodes [23, 24]. In the diagnosis of Meniere's disease, detailed anamnesis (clinical findings such as dizziness, hearing loss, and fullness at the ear) and examination are important. Some of the patients in this study had findings that requiredimaging and other examinations. Diplopia and dysarthria were determined in 6 patients and 4 patients complained of diplopia and paresthesia.In both clinics, the treatments for patients presenting with vertigo were in three main categories as symptomatic, specific and rehabilitation treatments. For this purpose, vestibular suppressants such as meclizine, dimenhydrinate, promethazineand diazepam and antiemetic drugs such as metoclopramide are used. For this purpose, anti-emetic drugs are used such as metoclopramide and vestibular suppressants such as meclizine, dimenhydrinate, promethazine and diazepam. Long-term use of drugs is not recommended and the formation of a normal compensation mechanism is preferred. Patients diagnosed with central vertigo in the otorhinolaryngology outpatient clinic are referred to theneurology department for the specific treatment. Although medical treatment for peripheral vertigo is given in the neurology outpatient clinic, patients are referred to the otorhinolaryngology outpatient clinic for rehabilitation. The Epley, Semont and Barbecue maneuvers are applied in patients with a specific diagnosis in the otorhinolaryngology outpatient clinic.

\section{CONCLUSION}

For patients presenting with the complaint of vertigo to the otorhinolaryngology and neurology outpatient clinics, a detailed anamnesis and a physical examination precede specific vestibular tests in the diagnosis. It was seen that the vast majority of patients that applied to both outpatient clinics had peripheral vertigo, especially BPPV. The use of maneuvers is promising in the BPPV treatment. A coordinated communication of both clinics is important for a rapid diagnosis and prevention of unnecessary examinations.

\section{Conflict of interest}

The authors disclosed no conflict of interest during the preparation or publication of this manuscript. 


\section{Financing}

The authors disclosed that they did not receive any grant during conduction or writing of this study.

\section{REFERENCES}

[1] Zwergal A, Möhwald K, Dieterich M. [Vertigo and dizziness in the emergency room]. Nervenarzt 2017;88:587-96. [Article in German]

[2] Choi JY, Lee SH, Kim JS. Central vertigo. Curr Opin Neurol 2018;31:81-9.

[3] Strupp M, Dieterich M, Brandt T. The treatment and natural course of peripheral and central vertigo. Dtsch Arztebl Int 2013;110:505-15.

[4] Tarnutzer AA, Berkowitz AL, Robinson KA, Hsieh YH, Newman-Toker DE. Does my dizzy patient have a stroke? A systematic review of bedside diagnosis in acute vestibular syndrome. CMAJ 2011;183:E571-92.

[5] Chawla N, Olshaker JS. Diagnosis and management of dizziness and vertigo. Med Clin North Am 2006;90:291-304.

[6] Berisavac II, Pavlović AM, Trajković JJZ, Sternic NM, Bumbasirevic LG. Drug treatment of vertigo in neurological disorders. Neurol India 2015;63: 933-9.

[7] Tusa RJ. Bedside assessment of the dizzy patient. Neurol Clin 2005;23:655-73.

[8] Neuhauser HK. The epidemiology of dizziness and vertigo. Handb Clin Neurol 2016;137:67-82.

[9] Havia M, Kentala E, Pyykkö I. Prevalence of Menière's disease in general population of Southern Finland. Otolaryngol Head Neck Surg 2005;133:762-8.

[10] Kerber KA, Meurer WJ, West BT, Fendrick AM. Dizziness presentations in U.S. emergency departments, 1995-2004. Acad Emerg Med 2008;15:744-50.

[11] Reis LR, Lameiras R, Cavilhas P, Escada P. Epidemiology of vertigo on hospital emergency. Acta Medica Portuguesa 2016;29:326-331.

[12] Lee H, Sohn SI, Cho YW, Lee SR, Ahn BH, Park BR, et al. Cerebellar infarction presenting isolated vertigo: frequency and vascular topographical patterns. Neurology 2006;67:1178-83.

[13] Narita S, Kurose M, Kobayashi K, Himi T. [Study on 242 in patients reporting vertigo and dizziness]. Nihon Jibiinkoka Gakkai Kaiho 2003;106:21-7. [Article in Japanese]

[14] Uno A, Nagai M, Sakata Y, Moriwaki K, Kato T. [Statistical observation of vertigo and dizziness patients]. Nihon Jibiinkoka Gakkai Kaiho 2001;104:1119-25. [Article in Japanese]

[15] Giudice R, Izzo R, Manzi MV, Pagnano G, Santoro M, Rao $\mathrm{MA}$, et al. Lifestyle-related risk factors, smoking status and cardiovascular disease. High Blood Press Cardiovasc Prev 2012;19:85-92.

[16] Neuhauser HK, Radtke A, von Brevern M, Lezius F, Feldmann M, Lempert T. Burden of dizziness and vertigo in the community. Arch Intern Med 2008;168:2118-24.

[17] Newman-Toker DE, Hsieh YH, Camargo CA, Pelletier AJ, Butchy GT, Edlow JA. Spectrum of dizziness visits to US emergency departments: cross-sectional analysis from a nationally representative sample. Mayo Clin Proc 2008;83:76575.

[18] Warach S, Gaa J, Siewert B, Wielopolski P, Edelman RR. Acute human stroke studied by whole brain echo planar diffusion-weighted magnetic resonance imaging. Ann Neurol 1995;37:231-41.

[19] Fiebach JB, Schellinger PD, Jansen O, Meyer M, Wilde P, Bender $\mathrm{J}$, et al. CT and diffusion-weighted MR imaging in randomized order: diffusion-weighted imaging results in higher accuracy and lower interrater variability in the diagnosis of hyperacute ischemic stroke. Stroke 2002;33:2206-10.

[20] Kattah JC, Talkad AV, Wang DZ, Hsieh YH, Newman-Toker DE. HINTS to diagnose stroke in the acute vestibular syndrome: three-step bedside oculomotor examination more sensitive than early MRI diffusion-weighted imaging. Stroke 2009;40:3504-10. [21] Brantberg K, Trees N, Baloh RW. Migraine-associated vertigo. Acta Otolaryngol 2005;125:276-9.

[22] Karatas M. Central vertigo and dizziness: epidemiology, differential diagnosis, and common causes. Neurologist 2008;14:355-64.

[23] Neuhauser H, Leopold M, vonBrevern M, Arnold G, Lempert $\mathrm{T}$. The inter relations of migraine, vertigo, and migrainous vertigo. Neurology 2001;56:436-41.

[24] Nelson JA, Viirre E. The clinical differentiation of cerebellar infarction from common vertigo syndromes. West J Emerg Med 2009;10:273-7. 